Dorneich, Michael C.,, Lancaster, Jeff A., Hamblin, Christopher J., DeMers, Robert E., and Olofinboba, Olu A. (2010). "Deriving Cursor Control Device Expectations for the Orion Crew Exploration Vehicle", Proceedings of the Human Factors and Ergonomics Society Conference, San Francisco, CA. September 27-October 1.

\title{
Deriving Cursor Control Device Expectations for the Orion Crew Exploration Vehicle
}

\author{
Michael C. Dorneich, Jeff A. Lancaster, Christopher J. Hamblin, Olu Olofinboba, and Robert E. DeMers, \\ Honeywell Laboratories
}

\begin{abstract}
A unique challenge for the Orion Crew Exploration Vehicle is the need for a novel cursor control device (CCD) that allows the crew to interact with display formats while seated and restrained. Display formats will contain "controllable elements" that will be used for input by astronauts, and will most likely not be laid out in a rectilinear grid. A four-way "caged" castle switch on the CCD was designed to travel only to controllable elements toward decreasing erroneous cursor movements. The ability of the four-way castle to intuitively navigate the cursor from a user perspective is a vital consideration. A cursor expectations study was conducted to understand dominant user expectations for CCD movements when controllable elements are not arranged on a rectilinear grid. Algorithms were developed that governed cursor movement in such a way as to match the dominant user expectations, to support the development of user mental models for cursor behavior, and to guide designers when laying out display formats.
\end{abstract}

\section{INTRODUCTION}

NASA's initiatives for advanced spacecraft development include the Orion Crew Exploration Vehicle (CEV), which is being designed as the nation's next generation human spaceflight vehicle to support future deep space missions. Orion is a capsule-type vehicle similar to, but significantly larger than, the Apollo spacecraft, and will carry up to four astronauts.

Orion will be equipped with a modern "glass cockpit" that will allow the astronauts to command and control all of the vehicle's systems from one of two operator stations. Unlike the Shuttle flight deck, Orion will have a fraction of the buttons, switches and dials that have traditionally been used in space vehicles. Instead, Orion's astronauts will monitor and command the vehicle's systems via graphics-based displays similar to those of modern flight decks. As this would imply, the design of Orion's displays and controls places an increased emphasis on human-computer interaction and usability. The importance of human factors is reflected in the fact that NASA, for the first time, has mandated usability and workload criteria as part of Orion's design requirements (Olofinboba, DeMers, Dorneich, Hamblin, and Wise, 2008).

Orion will require a controller that will allow the astronauts to interact with the vehicle during dynamic phases of flight (e.g., Launch, Entry) when the astronauts will be seated and restrained. The flight environment during these phases, in addition to the crew's personal protection devices, will limit the astronauts' reach and will thus severely impede their movement accuracy, thereby preventing them from directly interacting with the instrument panel. In an effort to provide Orion's crew with uninterrupted manual control of vehicle systems, a cursor control device (CCD) has been designed as part of Orion's avionics suite.

Perhaps the most influential requirement for the design of the CCD is to provide the crew the ability to manually override any automated functions that can be controlled within human capabilities. This includes allowing manual control of systems during the launch phases. This is not a trivial requirement as Orion's launch phases will subject the astronauts to gravitational and vibration loads greater than those experienced in either Apollo or Shuttle. In addition, the current geometry of the cockpit combined with the physical restraints of the crew's safety devices has induced several requirements for the CCD design.

One requirement that NASA and the Orion contractors worked closely to develop was a user interface that would allow operation of the vehicle by a single astronaut. Therefore, the CCD needed to provide the astronaut with unrestricted access to the avionics and their applications without impacting the GUI design.

Task and functional analyses also identified the need for a CCD to support remote interaction with the display formats. However, one outstanding question was the behavior of the cursor. The majority of display formats for Orion consist of system management pages that require user interaction with dozens of "controllable elements" to control various system elements on the vehicle. Given the extreme environments of use, a typical "free floating" cursor would not be appropriate. Thus, the decision was made to design a "caged" cursor that traveled only to controllable elements on the GUI in order to decrease erroneous cursor movements. However, it was unclear what the effects of the caged cursor would be on controllable element access time, or how restricted the cursor should be. An analysis (see Hamblin, DeMers, and Olofinboba, 2008) that considered functional, user, anthropomorphic, and ergonomic requirements resulted in a CCD design that included four controls: a four-way castle switch and three buttons, as shown in Figure 1.

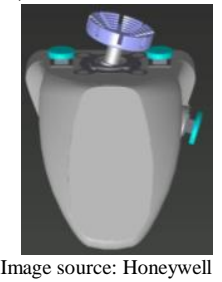

Figure 1. Orion Baseline CCD design.

Given the potential urgency with which the astronauts will need to navigate through the controllable elements (e.g., off-nominal or emergency operations requiring timely response), supporting the ability of astronauts to intuitively navigate the cursor is a vital consideration. One avenue with 
which to pursue this issue is to investigate what users would expect the cursor to do when actuated by the castle given the constrained (i.e., not "free floating") nature of the cursor movement. That is, the cursor movement will not be continuous. Instead, the cursor will "jump" from one controllable element to another. The location of the controllable elements will be governed by the task requirements of the display format. Therefore, the controllable elements will most likely not be laid out on a rectilinear grid. Notionally, a four-way castle switch has four cursor control movement directions (i.e., left, right, up, down). However, when controllable elements (or "nodes") are not in a rectilinear grid, the question arises of where the cursor jumps to when there are multiple diagonal options in the direction of cursor control movement.

\section{METHOD}

\section{Objective}

The objective of the cursor control movement study was to understand user expectations for cursor control movements when the nodes navigated are not on a rectilinear grid. Algorithms were then developed that governed cursor movement in such a way as to match the dominant user expectations.

\section{Participants and Tasks}

Eleven participants were given a paper survey in four trials. All the participants were engineers. The first three trials were constructed utilizing three notional Orion display formats. The fourth trial was not based on an Orion display format, but rather used to create some "special cases". The trials were constructed by removing all the graphics of the display format except for the controllable element themselves, resulting in an abstracted set of nodes (see Figure 2).

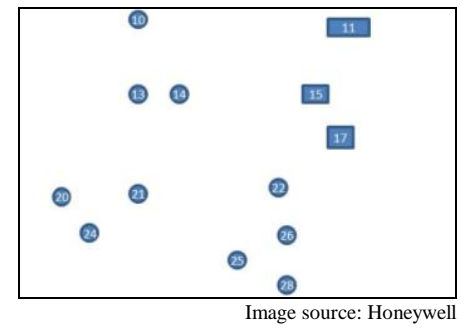

Figure 2. Example of controllable elements in a trial.

Basing trials on existing display formats was motivated by the desire to replicate the application domain as much as possible while still keeping the trials free of any visual items beyond the controllable elements that might drive cursor movement expectations.

\section{Procedure}

Participants filled out a "jump table" for left, right, up, and down cursor movements from each node (see Figure 3). Participants were given the following instructions:

1) Assume a device that has four inputs: left, right, up, and down. The device could be a gated cursor device, four arrow keys, or some other input device.
2) For each node on the display, indicate which node a left/right/up/down cursor control input would take you.

3) You may not be able to use every direction for a particular node.
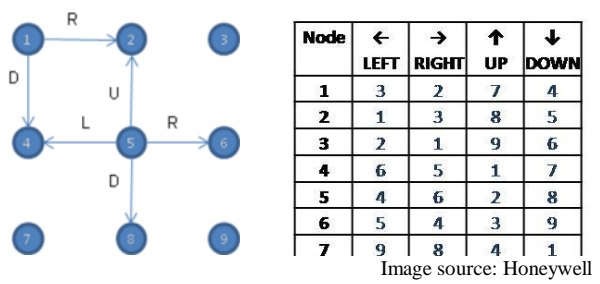

Figure 3. Sample trial and associated jump table.

\section{Data Analysis Terminology}

Prior to a description of the data analysis, a brief discussion of terminology is in order. Two conventions will describe the direction of the cursor control input versus the direction of the cursor control movement. As mentioned, the four-way CCD has four possible inputs - up, down, left, and right. The resulting movement can thus be likened to a northup map. That is, if the movement is straight up, the direction of the movement is defined as "north". The other three directions follow this format (see the left side of Figure 4).

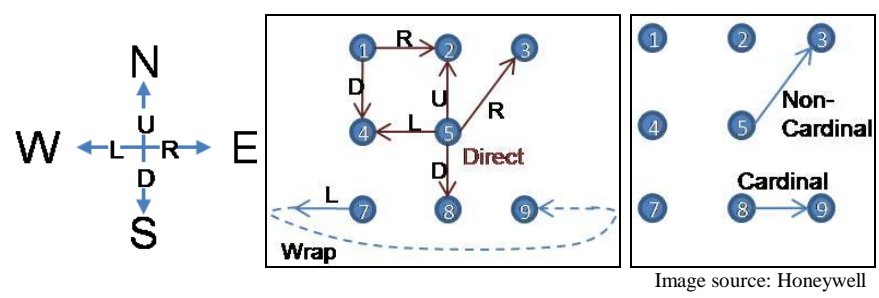

Figure 4. Nomenclature for input vs. movement direction (left). Examples of direct and a wrap transitions (middle), and a cardinal and a non-cardinal transition (right).

A direct transition is a cursor movement from one node to another within the boundaries of the display format (see Figure 4 middle). A wrap transition is a cursor movement from one node to another through an edge that "comes around" on a different side through the display boundary (see Figure 4 left). A cardinal transition is a cursor movement from one node to another where the two nodes align in the $\mathrm{x}$ or $\mathrm{y}$ axis (i.e., the transition is in the exact direction of the cursor input direction, e.g., north, east, west, south; see Figure 4 right). A non-cardinal transition is a cursor movement from one node to another where no cardinal option exists, and thus the movement is necessarily on a diagonal (see Figure 4 right).

\section{Data Analysis}

The data analysis plan included a combination of bottomup and top-down analyses. For top-down, the data were first assigned to one of four groups across two dimensions:

- Direct transitions vs. wrap transitions.

- Cardinal transitions vs. non-cardinal transitions.

For the bottom-up analysis, the data were analyzed by taking a 'question-based' approach within each group. That is, questions were asked of the data to determine how much of the observed results were explained by the answers. Different questions were asked until those whose answers explained the 
majority of the transitions that were identified. Example questions included:

- When a cardinal option existed, did participants choose it?

- For non-cardinal transitions, was there a preference of which diagonal to choose?

- Do Left/Right and Up/Down expectations differ?

- Are expectations different for wrapping than direct?

\section{Limitations}

There were two principal limitations to the study. Firstly, the trials did not systematically consider every possible distance/angle transition from a given node. A more systematic design would have created trials that presented an equal number of cases where the distance and angle deflection (from the intended cursor control movement direction) were varied to form a set of all possible transitions. The study that is described resulted from using notional displays as the basis for three of the four trials. It was felt that basing the trials on these notional displays outweighed the concern of exhaustively testing every possible transition combination.

Secondly, although the trials were based on notional display formats, all graphical elements except the controllable elements were removed. Some of the other graphics could have served to cluster nodes into a sub group, for instance, which may have driven expectations of where the cursor would jump to given a particular cursor control movement.

\section{RESULTS}

\section{Characterization of the data}

Across the four trials there were a total of 3025 total transitions, broken down into the following:

- 2253 Direct Transitions.

- 292 Wrap Transitions.

- 480 No Transitions.

The number of wrap transitions was low because only four participants provided any wrap transitions, perhaps because the instructions did not explicitly mention wrapping, so pnot all participants were aware that it was a possibility.

\section{Baseline results}

The data were analyzed to determine the most frequent response for each node's four transition possibilities. This provides, at a node-by-node transition level, a measure of consistency of the participants' answers. Considering the entire data set, $81.8 \%$ of the time (2085 of 2550 transitions) participants agreed with each other on a particular node transition, suggesting that across the four cursor control inputs, there was good and consistent agreement:

- LEFT: 542 of $646(83.9 \%)$

- RIGHT: 536 of $655(81.8 \%)$

- UP: 505627 of $(80.5 \%)$

- DOWN: 507 of 627 (80.9\%)

What this analysis revealed was that roughly 9 of the 11 participants chose the same transitions. In addition, there was good agreement for all four directions. It is worth noting those participants who chose a different node than the majority may still have chosen a node that most people would find acceptable - it just wasn't their first node of choice.

For purposes of the subsequent analysis regarding the effectiveness of the rule sets developed, the baseline results were used as the baseline for comparison. The implication was that if a rule set can be designed that matches the baseline performance, then the participants' dominant expectations have been identified.

\section{Cardinal transitions}

The first question to answer was what did participants do when a cardinal option existed? For instance, when a participant wanted to move right, and one of the possible target nodes was located directly east of the node, did that participant chose the direct transition, or another transition?

When a direct transition was available, $93.1 \%$ (580 of 623 ) of participants chose a cardinal LEFT/RIGHT when the option existed. However, when a cardinal UP/DOWN option existed, only $54.3 \%$ (202 of 281) of the participants chose it.

When a wrap cursor movement was made, only $27 \%$ (34 of 126) of participants chose the cardinal LEFT/RIGHT when the option existed. When a cardinal UP/DOWN option existed for a wrap cursor movement, $63.8 \%$ (30 of 47) chose it.

The results suggest that there was a strong preference amongst the participants for a direct LEFT/RIGHT cardinal cursor movement. However, there was no apparent preference for a direct UP/DOWN cardinal cursor movement. There was a strong preference against LEFT/RIGHT cardinal wrapping, and a weak preference for UP/DOWN cardinal wrapping.

\section{Up/down transitions}

So why did participants not always choose the cardinal UP/DOWN when it existed (since they preferred cardinal LEFT/RIGHT)? One possible explanation is that many cardinal options located north or south of a node were a far distance away, sometimes crossing imaginary rows (more on rows later). Thus, the next question to answer is what are the dominant expectations of UP/DOWN transitions (i.e. an up movement would look at nodes in the northern [upper] semicircle above the node). Data analysis revealed the following:

- $75.5 \%$ (438 of 580) of participants chose the closest direct node UP (see the left side of Error! Reference source not found.)

- $79.8 \%$ (474 of 594) of participants chose the closest direct node DOWN

In cases when two non-cardinal destination nodes were equally distant from the origin node, participants preferred the node with smaller relative angle deflection (from the cardinal direction). If they were the same distance/angle from the origin, participants preferred the left node (see Error!

Reference source not found. right).

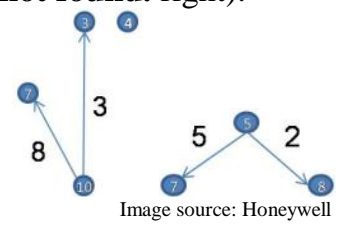

Figure 5. UP transition (left) - closer node was preferred over the cardinal; DOWN transition with equidistant nodes (right). 
The results suggest that UP/DOWN transition preference was dominated by distance. If two destination nodes were the same distance away, the preference was for the node with a smaller angle deflection. Finally, if the angles were the same, the left node was preferred.

\section{Wrapping Transitions}

Finally, why did participants not always choose a cardinal LEFT/RIGHT wrap when it existed? One possible explanation is that participants may have been viewing the wrapping in the context of a one-dimensional device design (i.e., rocker switch) where the cursor cycles through all nodes, wrapping to the beginning of the next line down when moving RIGHT from the last node in a row. Sometimes this is referred to as "typewriter wrapping" (see Figure 6). Data analysis revealed the following results, which suggest that LEFT/RIGHT wrapping was dominated by thinking in rows:

- $85.5 \%$ (53 of 62) wrapped to beginning of next line with RIGHT movement on right edge

- $79.4 \%$ (54 of 68) wrapped to end of previous line on LEFT movement at left edge node

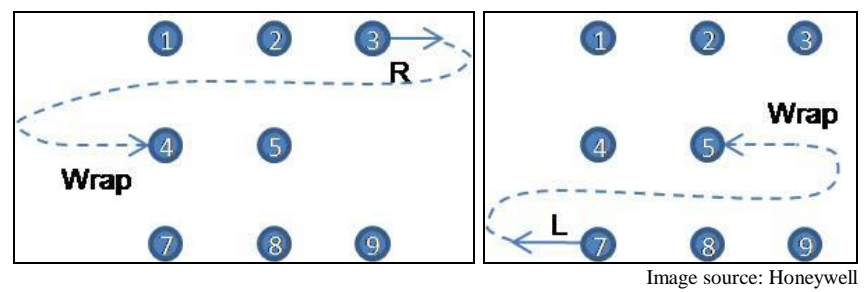

Figure 6. "Typewriter" wrapping

\section{RULE SETS}

Two alternate rules sets were developed to capture what was learned in the above data analysis. The rules were applied to the four trials, and the results were compared to the baseline to measure degree of fit. A high degree of fit would indicate that the rule set meets the dominant expectations for cursor movement. The rule sets may serve as a basis for algorithms with which to govern cursor response to operator inputs.

\section{Rule Set \#1: “Geometric Rules"}

This rule set is based on a completely "geometric perspective" when calculating the node to which to jump. For LEFT/RIGHT movements, when a cardinal option exists, choose it (see left side of Figure 7, transition $6 \mathrm{R} \rightarrow 7$ ). If no cardinal option exists, choose the diagonal with the closest distance (see left side of Figure 7, transition $6 \mathrm{R} \rightarrow 5$ ). If two diagonals are the same distance from the current node, choose the one with the smaller angle from the cardinal direction (transition $3 \mathrm{R} \rightarrow 1$ ). If the angles are equal, take the closest node UP (transition $4 \mathrm{R} \rightarrow 2$ ).
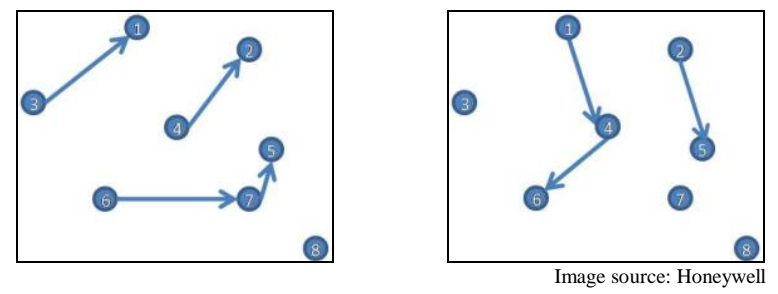

Figure 7. Rule Set \#1, LEFT/RIGHT direct (left figure) and UP/DOWN direct (right figure).

For UP/DOWN movements, choose the node with the closest distance within $+/-85^{\circ}$ (see right side of Figure 7, transition $1 \mathrm{D} \rightarrow 4$ ). Note that in the data, as the angle increased, the level of preference decreased as well. If two diagonals are the same distance from the current node, choose the smaller angle from the cardinal direction (transition $2 \mathrm{D} \rightarrow 5$ ). If the angles are the same, take the LEFT node (transition $4 \mathrm{D} \rightarrow 6$ ).

With respect to wrap transitions, 'edge nodes' must first be defined, either manually or automatically (algorithm to be determined). For the latter option, an example of a potential edge node algorithm would define edge node as having no other nodes within $+/-30^{\circ}$ in the direction of movement. Figure 8 illustrates Rule Set \#1 as applied to the LEFT and DOWN cursor inputs (direct transitions only) for trial A. The following rules thus apply to wrap transitions:

- For LEFT/RIGHT edge nodes, a RIGHT movement transitions to the left edge of the next row down, and a LEFT movement transitions to the right edge of the next row up (see "typewriter" wrapping of Figure 6).

- For UP/DOWN edge nodes, at the upper edge of the display format, an UP movement transitions to the lower edge node that is the smallest angle from the current node. At the lower edge of the display format, a DOWN movement transitions to the upper edge node that is the smallest angle from the current node.

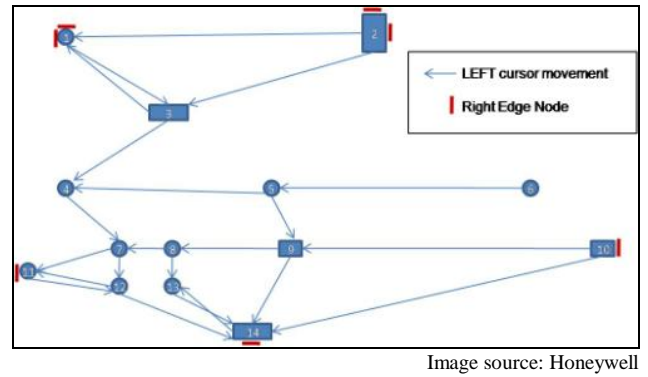

Figure 8. Rule Set \#1 as applied to Trial A (LEFT and DOWN).

Rule Set \#1 was applied to the data set to determine how much of the observed participant transition data could be explained. For Rule Set \#1, 77.1\% (1965 of 2550) of the observed transition results could be accounted for. For all cursor inputs, the following was observed:

- 79.1\% (511 of 646) LEFT transitions matched rule set.

- $77.7 \%$ (509 of 655) RIGHT transitions matched rule set.

- $75.6 \%$ (474 of 627) UP transitions matched rule set.

- 74.4\% (463 of 627) DOWN transitions matched rule set.

Overall, Rule Set \#1 has a $94.4 \%$ match with the Baseline. All four directions showed high agreement.

\section{Rule Set \#2: "Row Dominant"}

This rule set is based on a concept of pre-defined rows. A row is defined as any set of nodes arranged in a horizontal spacing where no two nodes share the same $x$ (horizontal) coordinates. Another way to visualize the definition of rows is to draw horizontal lines on the display that separate rows of nodes. Once these rows have been established, the transition rules are straightforward. 
Dorneich, Michael C.,, Lancaster, Jeff A., Hamblin, Christopher J., DeMers, Robert E., and Olofinboba, Olu A. (2010). "Deriving Cursor Control Device Expectations for the Orion Crew Exploration Vehicle", Proceedings of the Human Factors and Ergonomics Society Conference, San Francisco, CA. September 27-October 1.

A LEFT input results in a transition to the node with the next smaller $x$ within the row (i.e., immediately to the left within the row). A RIGHT input results in a transition to the node with the next greater $x$ within the row (i.e., immediately to the right within the row). An UP input results in a transition to the node with the shortest distance in the next higher row. A DOWN input results in a transition to the node with the shortest distance in the next lower row. Note that for both UP and DOWN inputs, if there are two nodes the same distance from the origin nose, choose the node with the smaller angle. If the two destination nodes are the same distance from the origin node and with the same angle, choose the one to the left.

Figure 9 illustrates these rules (direct only) applied to Trial C (only RIGHT and DOWN movements shown).

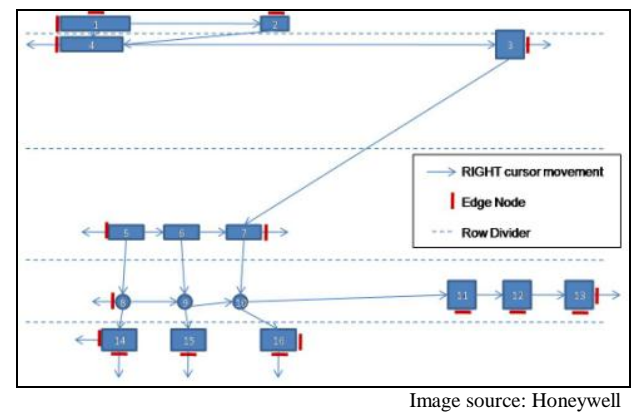

Figure 9. Rule Set \#2 applied to trial C (RIGHT and DOWN).

With respect to wrap transitions, edge nodes must be defined. All nodes on the top row represent upper edges; all nodes on the bottom row represent lower edges. Nodes in each row with the smallest $x$ (i.e., leftmost node in row) represent left edge nodes. Nodes in each row with the greatest $x$ (i.e., rightmost node in row) represent right edge nodes.

When executing a LEFT wrap, the transition is to the rightmost (smallest $x$ ) of the next upper row. For a RIGHT wrap, the transition is to the leftmost (greatest $x$ ) of the next lower row. When executing an UP wrap, the transition is to the smallest angle of the bottom row. When executing a DOWN wrap, the transition is to the smallest angle of the top row.

Rule Set \#2 was applied to the data set to determine how much of the observed participant transition data could be explained. For Rule Set \#2, 77.0\% (1963 of 2550) of the observed transition results could be accounted for. For all cursor inputs, the following was observed:

- $81.0 \%$ (523 of 646) LEFT transitions matched rule set.

- $78.0 \%$ (511 of 655) RIGHT transitions matched rule set.

- $75.1 \%$ (471 of 627) UP transitions matched rule set.

- $74.2 \%$ (465 of 627) DOWN transitions matched rule set.

Rule Set \#2 has a $94.2 \%$ match with the Baseline. In addition, all four directions showed high agreement.

\section{DISCUSSION}

It would be possible to apply the methodology outlined in the experiment above to fill in the cursor jump table in Figure 2. For each new display format, designers could poll a number of participants and accept as the "correct" cursor movement the most frequent response for each node jump. However, this is not a practical solution for a variety of reasons. Such a process would likely be quite time-consuming to apply to each new display format. Further, its application would not necessarily guarantee cursor transition consistency across displays. Finally, there would be no basis for a user to develop a consistent mental model with repeated use.

Rule Set \#1 has an advantage in that the rule set does not place any constraints on display format designers with respect to placement of commandable elements. There is no requirement to design in a grid pattern or a row pattern. Furthermore, since the transitions are based on a set of rules, they form the basis for the development of a mental model by users with repeated use. However, there are some disadvantages for this rule set as well. It is not clear that all nodes in any format design would be made 'reachable.' Further analysis would need to be conducted to provide evidence for the efficacy of this property in the rule set. Finally, this rule set is mathematics-based and, therefore, may not be the simplest solution to implement.

There are several advantages to Rule Set \#2. The use of this rule set makes it such that: 1) all nodes are reachable, and 2) all transitions are "undoable." That is, if a user mistakenly enters the wrong movement, doing the reverse of the last movement would return the cursor to the previous node, thereby supporting the desirable ability of user error recovery. In addition, the simplicity of the rule set makes it relatively easy for users to develop a mental model through repeated use. Finally, this rule set is relatively easy to apply to a given display format. Rule Set \#2 has the disadvantage that it does influence display format designers to lay out controllable elements in a quasi-row structure. A row structure is not strictly required, but the preference would be for easily identifiable rows of controllable elements. This may work well for system diagrams that are based on schematics, but may not work as well in flight control displays, where controllable elements are more randomly distributed within the display.

Given that both Rule Sets show a high degree of match with the baseline, it appears that either one could serve as a reasonable software algorithm to apply for governing four-way cursor movement. Rule Set \#2 is slightly preferred due to its simplicity of both application and use, as long as the requirement of a quasi row structure is not too restrictive to format designers.

\section{ACKNOWLEDGMENTS}

This paper was supported by a contract with Lockheed Martin (RH6-118204), for which Cleon Lacefield serves as the Program Manager of the Orion program. The opinions expressed herein are those of the authors and do not necessarily reflect the views of Lockheed Martin or NASA.

\section{REFERENCES}

Olofinboba, O., DeMers, R., Dorneich, M.C., Hamblin, C., and Wise, J. (2008), "A Systematic Tool for Deriving Crew Console Layouts," Proceedings of the Human Factors and Ergonomics Society's 52nd Conference, New York, NY, September 22-26.

Hamblin, C.J., DeMers, R.E., and Olofinboba, O.A. (2008), "Design of a Cursor Control Device for the Orion Crew Exploration Vehicle," Proceedings of the Human Factors and Ergonomics Society's 52nd Conference, New York, NY, September 22-26.

NASA. (2008). NASA-Orion Crew Exploration Vehicle. Available from NASA Web site: http://www.nasa.gov/mission_pages/constellation/orion/. 